\title{
12. Blockchain and merger control
}

\section{$1 \quad$ CHALLENGES}

In this first section, I introduce a typology of all blockchain concentrations. Some are technical by nature, as they concern the internal layers of two or more blockchains; while others are non-technical, as they concern the surrounding ecosystem.

\subsection{A Typology of Blockchain's Operations}

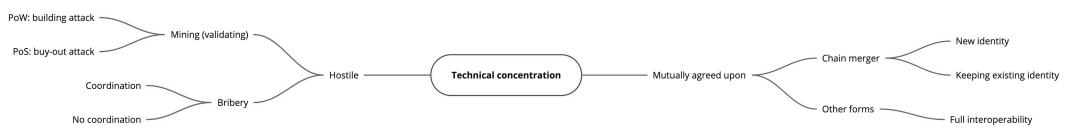

Figure 12.1 An overview of blockchain's concentrations

\subsubsection{Technical concentrations}

Blockchain concentrations may result from hostile takeovers. While these may look like proper acquisitions, I contend that the rules of merger control should not apply. On the contrary, when a blockchain concentration is mutually agreed upon, it might require a notification.

\subsubsection{Hostiles}

Hostile forms of blockchain concentrations - which we could also translate as forced takeovers - can be temporary or permanent. They are temporary when a blockchain nucleus takes "control" of a target Proof of Work blockchain for a specific period by devoting its computational power to it. ${ }^{1}$ Of course, the nucleus can use its existing computational power; but it may also rent or borrow the mining power from a third party so to reinforce the takeover.

1 Joseph Bonneau, "Hostile Blockchain Takeovers (Short Paper)," in Financial Cryptography and Data Security, eds. Aviv Zohar et al (Heidelberg, 2018) (describing this practice of "rental attack"). 
Permanent takeovers are also possible, but require more sophisticated strategies. The first of these strategies relates to mining or validating activity. If the target runs on Proof of Work, a "building attack" may amount to taking control of it by obtaining a permanent majority of the mining power. ${ }^{2}$ Where the target runs on Proof of Stake, a "buy-out attack" may be conducted by acquiring a majority of the existing capacity.

The second strategy is a "bribery attack." In this situation, a blockchain offers (manually or by smart contract) tokens to all participants of another blockchain that agree to burn their own (i.e., sending them to non-spendable addresses or specific smart contracts). ${ }^{4}$ When the vast majority of miners and users do so, one may consider that the takeover is complete. Prior coordination with miners and validators can help in completing such hostile takeovers - for example, by ensuring and explaining that the revenues from the mining activity will be higher in the acquiring blockchain. ${ }^{5}$ At the same time, disclosing the willingness to carry out a hostile takeover too far in advance could cause the operation to fail, as the target will have time to organize. ${ }^{6}$ Moreover, the more coordinated the strategy between blockchain participants, the more the operation will be considered mutually agreed upon.

In any case, one may question whether these hostile takeovers should be subjected to merger control. Those related to mining and validating activities are successful in the medium and long run only if the "acquired community" agrees to keep on mining or validating transactions in the chain. There are examples in which hostile takeovers have failed, and where blockchain communities have forked the blockchain after the takeover to seize back their own governance. ${ }^{7}$ Furthermore, in these "building attacks," there is no proper

2 Liehuang Zhu, et al., "Research on the Security of Blockchain Data: A Survey," Journal of Computer Science and Technology 35, no. 4, (2020) (describing the process).

3 Joseph Bonneau, "Hostile Blockchain Takeovers (Short Paper)," in Financial Cryptography and Data Security, eds. Aviv Zohar et al. (Heidelberg, 2018).

4 Andy Bromberg, "What the First Token Hostile Takeover Could Look Like," Medium, March 15, 2018, https://perma.cc/8JFE-FH3F.

5 Coordination with exchanges could also help, see Michael, "Altcoin Merger Season-Coming Spring 2019," November 4, 2018, https://perma.cc/AG35-D5AY.

6 Discussing how to prevent such hostile takeovers, see Andy Bromberg, "What the First Token Hostile Takeover Could Look Like," Medium, March 15, 2018, https:// perma.cc/8JFE-FH3F ("One way to apply this to this token hostile takeover would be to encode in the token's original smart contract that if a certain percentage of tokens outstanding are burned within a specific time period, those tokens are re-issued and distributed pro rata to all the remaining holders.")

7 Vitalik Buterin, "Coordination, Good and Bad," Vitalik Buterin's website, September 11, 2020, https://perma.cc/FBX5-R59E (explaining that forks are "a major and crucially important form of counter-coordination," preventing a few blockchain 
change in control of the entire blockchain, as only mining and validating activities are impacted.

As for hostile takeovers by way of bribery ("buy-out attacks"), they are successful only when the vast majority of users move to the other (more profitable) blockchain. These takeovers result from the interplay of competition. Anticompetitive practices could eventually be punished along the way, but in neither case is there a shift of power over the same entity (an essential criterion in merger control). Users are simply leaving for another blockchain. Hence, subjecting hostile takeovers to the obligation to notify, regardless of the procedural issues (i.e., their unpredictable and secret nature), would be nonsensical.

\subsubsection{Mutually agreed}

One may expect that mutually agreed concentrations will mainly take the form of "chainmergers," at least for the time being. ${ }^{8}$ Here, two blockchains merge their transaction histories and gather all new transactions in a single chain. The new entity may keep the identity of one of the two parents (the concentration would then be an acquisition), or it may also take on a new identity (the concentration would then be a merger). ${ }^{9}$ At least, that is the theory. In practice, however, these deals present several difficulties.

First, the two blockchains might not run on the same type of architecture. ${ }^{10}$ That being said, this technical problem will most likely end up with technical solutions. Second, the hard cap (if any) on the number of blockchain tokens must be maintained at equivalent levels. ${ }^{11}$ In other words, if one blockchain

users from colluding against the majority interest). For example, part of the Steem community - a social blockchain - has implemented a fork to escape a harmful collusion, see Paddy Baker, "Steem Hard Fork Confiscates \$6.3M, Community Immediately Takes It Back," CoinDesk, May 20, 2020, https://perma.cc/L6ZF-PDPH.

8 See Jonathan Jones, "JP Morgan Consider Blockchain Merger with Consensys," February 12, 2020, https://perma.cc/4HXB-BTVP. Also, discussing the possibility that setting up a blockchain consortium may be "subject to approval or at least scrutiny by merger control authorities," see Law Society and London Advocates, "Blockchain: Legal and Regulatory Guidance Report” (2020): 56.

9 For an example, see Yogita Khatri, "Polygon Acquires Hermez in \$250 million Deal that Includes First-Ever Token 'Merger'," The Block, August 13, 2021, https:// perma.cc/C34C-U34D (describing the acquisition of Hermez Network by Polygon and the merging of their native tokens).

10 Eric Lombrozo, "Consensus Rules - Changing Them Without Changing Bitcoin," YouTube, September 10, 2017, https://perma.cc/348B-BHRR ("We don't have a merge process in Bitcoin. There might be some future ideas eventually in other cryptocurrencies that might allow for merges but as Bitcoin stands right now, there really is no way to merge two chains that are incompatible").

11 Paddy Baker, "Chainmerger: Could BTC And BHC Ever Become One Again?" Crypto Briefing, September 24, 2018, https://perma.cc/GGB9-M3RA. 
has a cap of 20 million tokens and the other has a similar one, the merged blockchain should maintain that 20 million token cap instead of doubling it (its value would otherwise decrease dramatically). Further problems arise, for example, when one blockchain has a 20 million token cap while the other is limited at 3 million. Third, token holders would have to commit to burning their tokens to receive new ones. ${ }^{12}$ During this process, smart contracts could automatize the distribution of new tokens, depending on the number of old tokens being burned. This could also be done manually. ${ }^{13}$ Issuing tokens is called an "airdrop" where they are created out of thin air and distributed "for free" for a limited period of time, under specific conditions. The process could reward the adoption of the new blockchain - for example, by distributing 1.2 tokens per 1 burned token. ${ }^{14}$ These chainmergers also present challenges such as ensuring proper conversion, implementing the airdrop and verifying whether the tokens have been permanently burned before distributing new ones. Fourth, mechanisms would have to be put in place after the merger to convince the users remaining on one of the old chains to use the merged one instead. ${ }^{15}$ Let us recall that blockchains are immutable, and for that reason, one cannot erase them. They can only be abandoned.

Ultimately, mutually agreed concentrations will also take other forms than chainmergers - many of which are yet to be invented. For example, when two blockchains implement perfect interoperability, ensuring their protocols and operations are fully compatible, their ecosystems will be de facto combined into one (despite the continued presence of two entities). ${ }^{16}$ As a result, miners will mine blocks on either of these two blockchains without two core clients. This would achieve a concentration.

12 Chris Herd, "Why Blockchains Could Become Hostile Takeover Targets for Other Blockchains and How They Could Pull it Off," Medium, December 22, 2017, https://perma.cc/JD5H-EYQ2. One could also envision that they would transfer their old tokens to "the acquiring network's management team, which would then sell those tokens and take the proceeds," see Andy Bromberg, "Paying to be Bought: A Token Network Acquisition Blueprint," Medium, July 5, 2018, https://perma.cc/GT2T-HELB.

13 Michael, "Crypto to Crypto Merger-It's Possible!" Medium, December 11, 2018, https://perma.cc/KH93-BNV2.

14 Issues may arise if fewer of the new tokens are delivered compared to what had been promised.

15 Domocoin took over the Heptacoi, see Michael, "Crypto to Crypto MergerIt's Possible!" Medium, December 11, 2018, https://perma.cc/KH93-BNV2 ("The Domocoin $<>$ Heptacoin is the first recorded 'merger' between open source crypto projects (if there were others please let me know) and proves that M\&A is a viable growth hacking strategy for crypto projects.”)

16 Discussing interoperability in this context, see World Economic Forum, "Building Block chain(s) for a Better Plane" (2018), https://perma.cc/3FKJ-UDQS. 


\subsubsection{Non-technical concentrations}

Non-technical mergers do not affect the infrastructure or the governance of blockchains, ${ }^{17}$ but include the surrounding environment, such as media, exchanges, wallets, investment funds, hardware manufacturers, analytics firms, think-tanks and foundations. These operations create classic problems in terms of competitive analysis. For example, if a single exchange became so dominant that it captured 90 per cent of exchange activities, competition law concerns would logically arise. Also, mining companies could regroup to capture a larger share of the market. Finally, such concentrations could be vertical - for example, an exchange could acquire core developers.

On top of analyzing the relevant markets at stake, antitrust agencies should ensure that these transactions do not indirectly reduce the competitive pressure or lead to artificial centralization within blockchain markets. This will rarely be the case. Being outside of the blockchain ecosystem (such as I have presented in Chapter 4), these concentrations are unlikely to impact one or multiple blockchain layers. But the possibility cannot be entirely excluded, as outside operations could eventually impact blockchain's infrastructure. For example, when the incentive to invest knowledge, time or money in a blockchain is reduced because of a non-technical merger, this indicates the need to consider the competitive impact on the entire ecosystem.

\subsection{Evaluating Concentrations}

\subsubsection{Change in control}

\subsubsection{The logic}

In the United States, Section 7 of the Clayton Act (1914) governs mergers and acquisitions. It prohibits those transactions where "the effect of such acquisition may be substantially to lessen competition, or to tend to create a monopoly." On top of the Clayton Act, the Hart-Scott-Rodino Antitrust Improvements Act of 1976 ("HSR Act") gives the FTC and the DOJ Antitrust Division the power to review all operations concerning assets, non-corporate interests or voting securities.

Although none of the abovementioned texts is explicitly limited to transactions that involve a change of control, that element is central to the analysis. First, all acquisitions of less than 50 percent of a non-corporate entity are not reportable under the HSR Act. There is also an exemption for up to 10 percent of a corporation's voting securities. Furthermore, the element of control is

17 Nat Nead, "Tracking Reverse Mergers in Crypto \& Blockchain," Investment Bank, https://perma.cc/X7HZ-EA6T. 
essential when analyzing the "acquiring" and "acquired" persons. Put differently, two zones of control must be involved in the transaction and a transfer must be realized from one to another.

In Europe, the element of "control" is defined as the power to exercise decisive influence over an undertaking based on rights, contracts or other means. Control is characterized:

either separately or in combination and having regard to the considerations of fact or law involved, in particular by: (a) ownership or the right to use all or part of the assets of an undertaking; (b) rights or contracts which confer decisive influence ... ${ }^{18}$

All concentrations involving a lasting change of control must be notified. The change can be de jure or de facto; it can include negative (e.g., veto rights) or positive (e.g., voting rights) capacities. Measuring that transfer is challenging when it comes to blockchain.

\subsubsection{In practice}

As I have shown, distinct forms of horizontal control are exercised within blockchains, as opposed to the firm, where control is vertical by nature. This makes pinpointing who controls the blockchain more complex.

As a matter of fact, there are few assets linked to a blockchain. Acquiring a blockchain means acquiring its key usage (miners and users), and its expertise (core developers). To be sure, blockchain concentrations can take different forms, but they all lead to a similar result: merging two blockchain nuclei into one. In other words, a takeover is complete whenever one blockchain nucleus ensures the survival of another blockchain. When the nucleus participants refuse to do so, their blockchain conserves the means to survive and keeps on exerting competitive pressure on the others. Participants' behaviors are central to the analysis for that reason.

Identifying the behavioral factors that suggest a possible change in control will help agencies. As I have explained, the participants in the nucleus have the power to influence the blockchain. By collaborating and therefore evading (some of) the constraints imposed by others, they may decide to acquire another blockchain. For instance, a nucleus could start an airdrop and set up a smart contract to offer freshly minted tokens to the users of another blockchain. One nucleus could also agree with another to start a fork with the ambition of joining the two chains.

They could also achieve their objective by setting up sufficiently high incentives for the operation to succeed, as they cannot impose the takeover on

18 European Commission, "EC Merger Regulation Guidelines," Council Regulation (EC) No 139/2004. 
other members (Chapter 7 explains how members of the nucleus can impose these strategies). These apply to both public permissionless blockchains and private ones.

In the end, agencies will have to decide which mechanisms are sufficiently robust and effective to incite blockchain nuclei to approve and complete takeovers. Doing so will help agencies identify which actions could succeed and, therefore, trigger the need for prior notification. This is no easy task, but it is a crucial one. Agencies do not want to impose notifications in situations where the incentives are not good enough, nor do they want to do so once the concentrations have been completed. Meeting this challenge will require agencies to carefully analyze existing blockchain code and ecosystems in search of answers.

\subsubsection{Analyzing thresholds}

Not all operations that involve a change of control are subject to a notification requirement. They must also pass relevant thresholds. In both the United States and Europe, these thresholds are linked to the turnover of the entities involved in a transaction. Transposing these thresholds to both public permissionless and private blockchains will, once again, prove challenging. Determining the (representative) turnover of these blockchains, as I have explained, is difficult. Indeed, their value is initially expressed in their native tokens. One can certainly convert these into fiat currencies and one can calculate their average over a year; but this would negate their numerous fluctuations.

Simply converting tokens to express a blockchain's turnover could end up in a situation where agencies might find that one blockchain enjoys significant market power because its turnover, converted into dollars or euros, is high on average, despite substantial fluctuations. On the contrary, agencies might find that another blockchain enjoys lesser market power because its average turnover, once converted, is lower despite its stability. This situation would paint an erroneous picture of the market reality. A blockchain whose value soars for a few months before collapsing does not necessarily benefit from significant market power. Therefore, new analyses must supplement the metrics that are currently used. The second section of this chapter deals with this question.

\section{OPPORTUNITIES}

Despite the challenges blockchain creates for the control of concentrations, it also opens up opportunities. Embracing the advent of blockchain ecosystems will notably lead antitrust agencies to adopt a more technological approach to merger analysis and modernize existing procedures. 


\subsection{A Technological Approach}

\subsubsection{Analyzing technology (for real)}

Analyzing blockchain concentrations requires a good understanding of the technology. Acquiring this knowledge is a challenge as much as an opportunity to modernize merger control. ${ }^{19}$ That applies to notifications and assessments of anticompetitive effects.

Most notifications are triggered by a change in legal structure (e.g., the acquisition of shares), but one may also take on legal instruments (e.g., contracts) to analyze the acquisition of de facto control. Such analyses neglect the intention behind the operation; while, as I have shown in the first section, the concentration of public permissionless blockchains is a matter of implementing the proper incentives. Blockchain concentration will thus require more from agencies - namely, to study not only how blockchain code is used or changed for the purpose of an operation, but also the effects of these changes (i.e., if they create sufficient incentives or not).

Assessing the competitive effects of blockchain concentrations also requires agencies to understand the underlying technology. Merely looking at market shares will be insufficient. Antitrust agencies occasionally look at other metrics, such as competitors' size, the number of recent entries and so on. But those are poor substitutes for analyzing the technology at stake in digital mergers. Code and programming may reveal some potential for growth, compatibility diversification outside one relevant market, network effects and potential consumer lock-in (or absence thereof).

Put differently, analyzing blockchain's code will allow agencies to assess its dynamic capabilities ${ }^{20}$ - that is, the capacity to absorb and integrate external knowledge. In fact, one may talk about dynamic technical capacity - that is, the ability to develop new technical capability on top of or using an existing technology. ${ }^{21}$ That is crucial in assessing dynamic competition and monopoly

19 Robert Zev Mahari, Sandro Claudio Lera and Alex Pentland, "Time for a New Antitrust Era: Refocusing Antitrust Law to Invigorate Competition in the 21st Century," Stanford Journal of Computational Antitrust 1 (2021): 57 (showing how technological analysis can inform antitrust agencies in merger control).

20 David J. Teece, Gary Pisano and Amy Shuen, "Dynamic Capabilities and Strategic Management," Strategic Management Journal 18, no. 7 (1997), https://perma .cc/DVE6-8QMF?type=image.

21 Mary Tripsas, "Surviving Radical Technological Change through Dynamic Capability: Evidence from the Typesetter Industry," Industrial and Corporate Change 6, no. 2 (1997). As I explained (see Chapter 10), Ethereum's code is Turing complete, meaning that one can use its Virtual Machine to compute anything computable. Bitcoin's current code is not Turing complete, which provides Bitcoin's blockchain with less dynamic technical capacity. 
power. ${ }^{22}$ This will ultimately generate enough expertise to update merger control guidelines and institutionalize a technological assessment both for blockchain and outside.

\subsubsection{Blockchain unicorns and killer acquisitions}

The subject of killer acquisitions has become increasingly important to antitrust policy debates. In the absence of empirical studies documenting the frequency of the phenomenon, let me simply explain what is at stake. A "killer acquisition" is the purchase of a company by a competitor to end its products or services, where the costs of the acquisition are recovered by the absence of competition.

Part of the antitrust academic community has made the point that most of these acquisitions escape merger control. Indeed, only concentrations above a certain threshold must be notified. In the United States and Europe, these thresholds concern at least two entities involved in the operation - typically the acquirer and the target. When a company acquires an emergent (and small) competitor, the operation is likely to fall below the threshold. Austria and Germany have introduced alternative thresholds linked to the value of the transaction to remedy this issue - the idea being that the acquisition of a small competitor for several hundred million euros is something that antitrust agencies may want to study.

When it comes to the blockchain industry, one could argue that the price of setting up the right incentives equals the value of the transaction. But that value goes directly to the blockchain's participants, not to a handful of owners, as is the case with firms. For this reason, one should not be too worried about killer acquisitions - the purchase of dynamic blockchain unicorns by other blockchains or firms - within the blockchain sphere, as it takes more than convincing a few individuals to sell. Considering how unlikely it is that the vast majority of participants would agree to a killer acquisition, the price that an acquirer would have to pay to compensate each of them and the fact that blockchain's participants could reactivate their blockchain should the acquisition turn out to be for "killing" purposes, the priority for antitrust agencies seems to be elsewhere. Blockchain escapes this issue thanks to the lack of centralized control.

22 That importance is increasing, as most of today's new technologies result from "new combinations" of past technologies, see W. Brian Arthur, The Nature of Technology: What it Is and How it Evolves (Free Press, 2009) (explaining that "technologies ... arose as combinations of other technologies"). 


\subsection{Modernizing Procedures}

\subsubsection{Improving assessments}

Blockchains can facilitate the exchange of pre-transaction information between parties. This can be done in two ways. The first is to use blockchains as a trusted database. M\&A transactions involve due diligence, which is lengthy and costly - the target provides information to the potential acquirer to assess the risks associated with the acquisition. Private blockchains could be used in that regard to replace data rooms, as they are more secure. ${ }^{23}$ Specific rights of access could be granted, with or without editing rights. The second is to create smart contracts that verify information or automate transactions. ${ }^{24}$

Blockchains can also facilitate exchanges between the parties and antitrust agencies. They could notably be used to standardize the information agencies require from the parties or to facilitate the transfer of complete and verified information. One could imagine a world where companies and antitrust agencies use blockchains to facilitate the analysis of operations. Antitrust agencies currently suffer from the incompleteness of the information they receive. ${ }^{25}$ Blockchain could assist agencies should they require companies to document their market shares into private blockchains. Deleting information from these blockchains - for instance right before a merger - would leave a trace in the ledger.

One may also question whether the information provided by the parties is always accurate, if only because they must estimate the market shares of their competitors. To remedy this issue, agencies could require companies to centralize all information they send to various administrations in private blockchains. Antitrust agencies would then obtain a more comprehensive view of markets. They would be able to cross-reference different sets of data and, in the end, get a clearer view of the industry.

23 SS\&C, “Blockchain in M\&A: Hope or Hype?” December 4, 2018, https://perma .cc/A83B-W3MQ.

24 Ataullah Khan, "How Mergers and Acquisitions Could Occur on the Blockchain," Hackernoon, March 2, 2018, https://perma.cc/5PV2-Q2PK; "Blockchain \& the Future of M\&A," The Non Executive, June 2, 2020, https://perma.cc/VD45-KXCQ.

25 See Olympic/Aegean Airlines, Case COMP/M.6976, C (2011)316 (EC, 2011) (underlining the "poor quality, incomplete and/or inaccurate" information, because the ferry operators' databases were "not as developed as the sophisticated systems/ databases used by airlines.") See also Ryanair/Aer Lingus, Case No COMP/M.4439, $\mathrm{C}(2007) 3104$ (EC, 2007) in which the Commission explained that it did not have sufficient information regarding several routes. More recently, see Merck/Sigma-Aldrich, COMP/M.8181, to be published (EC, 2021) ("Not only was the project not disclosed and discussed in remedy submissions, but information about it was also witheld in replies to specific requests for information"). 
Agencies could also use blockchains to centralize their data in a single shared space. Access (but not necessarily writing rights) to one agency's private blockchain could be granted to another one - for example, if a company is simultaneously filing a merger in two jurisdictions. Access to an agency's blockchain could also help when several proceedings follow one after another over time, as blockchain immutability ensures that records cannot be altered (whether voluntarily or involuntarily). This immutability would strengthen the trust agencies have in each other and would ultimately allow better-informed analysis of merger proceedings and anticompetitive practices.

Smart contracts could also speed up procedures by automating the request of additional information based on that provided by the parties. This would avoid a considerable amount of back and forth. And should the agencies be comfortable enough with the technology, smart contracts could force parties to implement specific commitments - for example, by automating divestitures in case of noncompliance with behavioral remedies. That being said, smart contracts will most likely be used not only to ensure better monitoring of obligations, but also to survey all the firms' behaviors post-transaction. Data about the new entity could be automatically transmitted to antitrust agencies, which would considerably enhance their knowledge of the market.

In sum, blockchain could provide antitrust agencies with better information and will simplify interactions with companies. Today, a significant amount of data is sent by the parties (e.g., the relevant market shares) in the form of unverifiable Excel sheets. Monitoring is difficult. Blockchain's capacity to ease merger procedures will surely be explored for these reasons.

Let us also note (if I may take this little detour) that blockchain will help antitrust agencies in other fields than merger control. First, blockchain will most certainly be used to identify nascent practices. Antitrust agencies continuously point out the difficulty of detecting anticompetitive practices because their databases are incomplete. ${ }^{26}$ Public permissionless blockchains could provide a remedy - at least a partial one - to this difficulty by enabling antitrust agencies to monitor final prices to consumers and detect anticompetitive trends. ${ }^{27}$ This will lead them to discern patterns and thus increase their knowledge of the cycles in which anticompetitive practices are committed

26 Underlining that "not all facts can be observed or measured with high accuracy and most datasets are incomplete or otherwise imperfect," European Commission, "Best Practices For The Submission Of Economic Evidence And Data Collection In Cases Concerning The Application Of Articles 101 And 102 TFUE And In Merger Cases," Staff Working Papers (2011), https://perma.cc/675N-7374.

27 OECD, "Executive Summary of the Hearing on Blockchain and Competition Policy," DAF/COMP/M(2018)1/ANN8/FINAL (2019): 3, https://perma.cc/L2F8 -JXKY. 
(this process is often referred to as "cascade analysis"). Second, blockchains could also speed up and improve the accuracy of proceedings once a practice has been identified. Antitrust agencies could ask incriminated companies to provide specific information by way of smart contracts.

\subsubsection{Improved monitoring}

Blockchains could help enforcers monitor companies' behavior - specifically, when they impose remedies and commitments that seek to restore competition on the merits (anticompetitive cases) or ensure that markets will remain competitive (in merger cases). ${ }^{28}$ In either case, agencies need to identify effective solutions that they can follow up on. Here again, blockchain could help.

As far as the design of the commitments and remedies is concerned, one could use smart contracts to ensure that they are correctly implemented. ${ }^{29}$ Structural commitments could be set up by way of smart contracts so that they are activated only under certain conditions. ${ }^{30}$ For example, one could force a company to sell specific assets or undertakings if a product's price exceeds a pre-defined level. Smart contracts could also improve behavioral commitments. These usually take the form of an obligation to provide access to an essential facility or intellectual property rights; ${ }^{31}$ smart contracts could enable agencies to ensure that these are well observed.

Other smart contracts could help in implementing more detailed obligations. A smart contract could combine behavioral and structural commitments, activating one depending on the other. For example, a smart contract could require prices to be kept below a certain level; otherwise antitrust agencies would receive a signal and another smart contract would trigger structural remedies. And without going that far, one may expect that companies will observe

28 The implementation of remedies can be observed with regard to both ex post conduct through Articles 7 and 9 of Council Regulation 1/2003, 2003 O.J. (L 1) 46 and ex ante conduct pursuant to Articles 6(2) and 8(2) of Council Regulation 139/2004, 2004 O. J. (L24) 29.

29 Generally speaking, the European Commission prefers behavioral commitments to address anti-competitive practices and structural remedies in merger control, see Benjamin Lörtscher and Frank P. Maier-Rigaud, "On the Consistency of the European Commission's Remedies Practice," in Substance, Process and Policy, eds. Damien Gerard et al. (Wolters Kluwer, 2019): 2.

30 See Chris Pike, OECD, "Blockchain Technology and Competition Policy," DAF/ COMP/WD(2018)47 (2018): 9, https://perma.cc/MGJ5-NH7Y (discussing the potential "cost reduction effect" using smart contracts).

31 Generally, behavioral remedies take the form of access remedies, interoperability obligations and internal remedies such as firewalls and corporate governance measures. 
stricter compliance, since smart contracts increase antitrust agencies' ability to monitor commitments. ${ }^{32}$

But before this happens, several challenges will be encountered along the way. First, implementing smart contracts will require (some) familiarity with code, although several services are being developed to allow easy smart contract design (called "no code"). A psychological barrier could nonetheless exist. Second, smart contracts are unstoppable, and accordingly, they may create disparities with the actual world in case of unforeseen events. Third, smart contracts are written in computer languages. This has advantages and limits, as it provides companies with some form of (extreme) certainty but reduces flexibility. Finally, one may be concerned that public blockchains could split or be altered by majority votes, and that all smart contracts would be extinguished. The use of private blockchains run by agencies seems safer in that regard.

\section{CHAPTER SUMMARY AND BEYOND}

In this chapter, I have studied all the different forms of concentrations that may occur in the blockchain ecosystem and in relation thereto. I have shown that technical concentrations can be hostile - whether they result from a "building attack," in which a majority of the mining power is captured, or from a "bribery attack," in which the participants are incentivized to move to another blockchain. These operations should not, however, be subjected to notification requirements, as they generally result from the interplay of competition and entail no change of control. At most, they might sometimes amount to anticompetitive practices.

Things are different with mutually agreed concentrations. They mainly take the form of chainmergers, where two blockchains or more merge their entire transaction histories. I have shown that these concentrations raise many practical difficulties, yet remain likely to happen. Then, I moved on to the study of non-technical concentrations - that is, all operations concerning the blockchain environment, but not blockchain itself. I explained that existing merger control rules more easily capture these concentrations, and that agencies should pay attention to their impact on the chain.

After exploring these different forms of concentrations, I studied the two main criteria of merger control: the change in control and the relevant transaction thresholds. I explained that because blockchains - public permissionless ones and most private ones - exert no coercive power over their participants,

32 Ajinkya M. Tulpule, "Enforcement and Compliance in a Blockchain(ed) World," CPI Antitrust Chronicle 1 (2017). 
one blockchain can take control of another only when the participants consent to the operation. Here, I argued that agencies should study which incentives are convincing enough for participants to agree to the takeover. As for the threshold requirements, I explained that one cannot rely on blockchains' turnover expressed in native tokens that poorly represent their market power.

The first section of the chapter led me to conclude that a more technical analysis is required. This was further discussed in the second section. I started by proposing that analysis of the underlying technology is essential for antitrust enforcers. It helps not only the notification process, but also the assessment of these operations. Indeed, antitrust agencies will be better equipped to understand competitive forces if they understand the technology's capacity to generate network effects, mutate, be combined and so on. The analysis of the technology reveals, for example, that killer acquisitions are unlikely to happen in the blockchain ecosystem.

Lastly, this technological approach could also be used to modernize merger control procedures. Blockchain could help standardize the process and give agencies access to more complete sets of data. The use of smart contracts could also require specific information to be placed on the ledger to improve the monitoring of remedies and commitments. And smart contracts would enable parties and agencies to craft more accurate and complete remedies. In a nutshell, these are the benefits of the cooperation between law and technology, on top of those I have already described when dealing with anticompetitive practices. In Part 3 of this book, I explore how this collaboration can be fostered. 